\title{
Resonant scattering of positronium as a quasifree electron
}

\author{
M. Shipman, ${ }^{1}$ S. J. Brawley, ${ }^{1}$ L. Sarkadi, ${ }^{2}$ and G. Laricchia ${ }^{1, *}$ \\ ${ }^{1}$ UCL Department of Physics and Astronomy, University College London, Gower Street, London WC1E 6BT, United Kingdom \\ ${ }^{2}$ Institute for Nuclear Research of the Hungarian Academy of Sciences (ATOMKI), Pf. 51, H-4001 Debrecen, Hungary
}

(Received 5 December 2016; published 21 March 2017)

\begin{abstract}
In order to clarify the physics underlying the observations of the electronlike behavior of positronium (Ps) and its resonant scattering from $\mathrm{CO}_{2}$, we have measured the $\mathrm{Ps}+\mathrm{N}_{2}$ total cross section and found it also to exhibit significant structure. Analysis of the resonances reveals that Ps is distorted in the collisions and classical trajectory Monte Carlo calculations indicate that the electron is on average closer to the target than the positron, which may in turn bind resonantly to the ensuing temporary negative ion. This description of the nature of Ps resonances agrees with long-standing theoretical predictions.
\end{abstract}

DOI: 10.1103/PhysRevA.95.032704

Positronium (Ps) is the bound state of an electron $e^{-}$ and a positron $e^{+}$. It is the lightest known atom, electromagnetically bound by $6.8 \mathrm{eV}$, half the energy binding the electron and proton in atomic hydrogen. Being composed of a particle and its antiparticle, it is intrinsically unstable and annihilates emitting characteristic $\gamma$ rays, which can provide useful information in galactic astrophysics (see, e.g., [1]), material and surface physics (see, e.g., [2,3]), and medical applications (see, e.g., [4,5]). From a more fundamental physics perspective, challenges include the production of a Bose-Einstein condensate of Ps [6,7] and the test of its gravitational interaction with Earth [8,9].

The lifetime of Ps depends on its spin, the triplet or orthopositronium ( $o$-Ps) being longer lived $(\simeq 142 \mathrm{~ns}$ for $\left.1{ }^{3} S_{1}\right)$. In the past couple of decades, a beam of $o$-Ps has enabled investigations of its scattering from a variety of atomic and molecular targets in the single-collision regime and at well-defined energies over the range $\simeq 1-400 \mathrm{eV}$, complementing a significant body of theoretical work [10,11]. Experimentally, the total and fragmentation cross sections have been determined [11-16], the former revealing a strong similarity to the cross sections for equivelocity electron projectiles $[11,15,16]$. Expecting that Ps scattering would be a coherent sum of electron and positron scattering [17], the implied dominance of the electron was surprising $[15,18]$. It guided the choice of $\mathrm{CO}_{2}$ as a target because of the prominent ${ }^{2} \Pi_{u}$ shape resonance occurring for electron projectiles at velocities around 0.5 a.u. $\left(\simeq 1.09 \times 10^{6} \mathrm{~m} \mathrm{~s}^{-1}\right)$, leading to the first observation of resonant scattering of Ps [15].

The first prediction of a resonant collision for Ps arose from the early elaborate treatment of $s$-wave scattering of $\operatorname{Ps}(1 s)+\mathrm{H}(1 s)$ by Drachman and Houston [19]. In the cross section associated with the singlet state of the two electrons, they found a peak near $4.45 \mathrm{eV}$ corresponding to unstable states of the positron trapped in the field of the $\mathrm{H}^{-}$ion [19-21].

\footnotetext{
*Corresponding author: g.laricchia@ucl.ac.uk

Published by the American Physical Society under the terms of the Creative Commons Attribution 4.0 International license. Further distribution of this work must maintain attribution to the author(s) and the published article's title, journal citation, and DOI.
}

The prediction of an infinite Rydberg series of resonant $e^{+} \mathrm{H}^{-}$ states (converging to the $\mathrm{H}^{-}$threshold at $6.05 \mathrm{eV}$ ) has been confirmed by sophisticated coupled-pseudostate calculations, which include an explicit representation of $e^{+}+\mathrm{H}^{-}[22,23]$. Resonant states of $\mathrm{PsH}$, in which the positron binds to the doubly excited resonant $2 s^{2}{ }^{1} S^{e}$ state of $\mathrm{H}^{-}$, have also been theoretically studied [24].

In an attempt to clarify the physics underlying the observations of Brawley et al. [11,15,16], we measured the Ps total cross section $Q_{\mathrm{T}}^{\mathrm{Ps}}$ for $\mathrm{N}_{2}$, which displays a resonant peak in its electron total cross sections $Q_{\mathrm{T}}^{-}$at velocities that have recently become accessible with the equipment at UCL $[11,25]$. Once again, we have found $Q_{\mathrm{T}}^{\mathrm{Ps}}$ to display a structure near that occurring in $Q_{\mathrm{T}}^{-}$. Analysis of the data for both $\mathrm{CO}_{2}$ and $\mathrm{N}_{2}$ indicates that Ps is strongly polarized in the collision and that this distortion gives rise to its electronlike scattering. The positions of the Ps resonances, relative to those for electrons, are used to quantify the Ps distortion.

The beamline at UCL has been described in detail elsewhere (see, e.g., [11,15,26]). Slow positrons are obtained from a ${ }^{22} \mathrm{Na}$ source covered with a solid rare-gas moderator (krypton or neon), accelerated to the required energy by a positive potential applied directly to the source, and radially confined by an axial magnetic field from the moderator to the scattering region. Ps is produced via the charge exchange reaction from a gas $X, e^{+}+X \rightarrow \mathrm{Ps}+X^{+}$[27]. In this work, $\mathrm{H}_{2}$ was used as the production gas [28] except at the lowest energies where Ar was used [25]. The kinetic energy of the Ps beam $E_{\mathrm{Ps}}=E_{+}-I+B$, where $E_{+}$is the positron beam energy, $I$ the first ionization energy of the target, and $B$ the binding energy of the Ps atom $\left(6.8 \mathrm{eV} / n^{2}\right.$, with $n$ its principal quantum number) [29]. The energy spread of the Ps beam essentially corresponds to that of the primary $e^{+}$beam, which depends on the moderator $\left(\Delta E_{+} \sim 1.0 \mathrm{eV}\right.$ for solid $\mathrm{Ne}$ and $\Delta E_{+} \sim 1.7 \mathrm{eV}$ for solid $\mathrm{Kr}$ ) [14,25,30-32]. The transmitted positrons are reflected electrostatically while Ps enters a second scattering cell of effective length $\ell_{\text {eff }}$ containing the target gas. Signals from a channel electron multiplier or multiplier array at the end of the beam line are counted in coincidence with those from surrounding $\gamma$-ray detectors. The $Q_{\mathrm{T}}^{\mathrm{Ps}}$ values are determined using the Beer-Lambert law $I=I_{0} \exp \left(-p \ell_{\mathrm{eff}} Q_{\mathrm{T}}^{\mathrm{Ps}} / k T\right)$, by measuring $I_{0}$ and $I$ (the incident and transmitted Ps beam intensities) and $T$ and $p$ (the temperature and pressure of the target gas), with $k$ being the Boltzmann constant. 


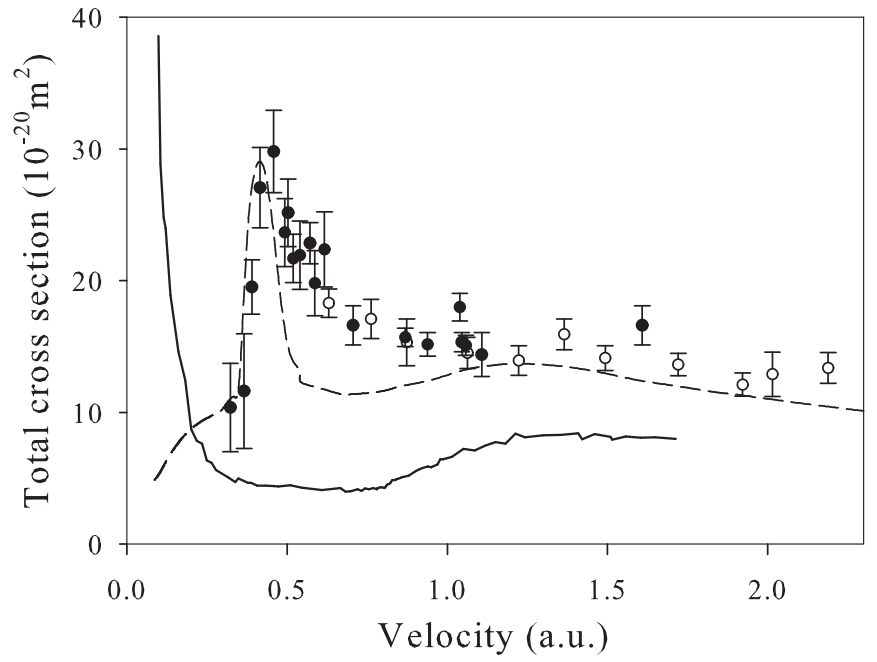

FIG. 1. Total cross sections for equivelocity Ps, positrons, and electrons colliding with $\mathrm{N}_{2}$. Here the $\bullet$ represent the $Q_{\mathrm{T}}^{\mathrm{Ps}}$ measurements from the present work and the $\circ$ those from [16], while shows the corresponding $Q_{\mathrm{T}}^{+}$from [36] and --- the $Q_{\mathrm{T}}^{-}$ from [33].

The present measurements of $Q_{\mathrm{T}}^{\mathrm{Ps}}$ for $\mathrm{N}_{2}$ are plotted as a function of the projectile velocity in Fig. 1 and are compared with previous results [16], $Q_{\mathrm{T}}^{-}$, and the corresponding positron-impact cross sections $Q_{\mathrm{T}}^{+}$[33-36]. Once again, in agreement with previous work $[15,16], Q_{\mathrm{T}}^{\text {Ps }}$ is very close to $Q_{\mathrm{T}}^{-}$ when compared at the same projectile velocity. The peak in $Q_{\mathrm{T}}^{-}$for $\mathrm{N}_{2}$ at 0.42 a.u. corresponds to the ${ }^{2} \Pi_{g}$ shape resonance (see, e.g., [33] and references therein); in $Q_{\mathrm{T}}^{\mathrm{Ps}}$, a maximum of similar magnitude $\left[(29.8 \pm 3.1) \times 10^{-20} \mathrm{~m}^{2}\right]$ is observed at a slightly higher velocity ( $\simeq 0.46$ a.u.).

The similarity between $Q_{\mathrm{T}}^{\mathrm{Ps}}$ and $Q_{\mathrm{T}}^{-}$for a given target at the same projectile velocity [16] and the occurrence of resonances in Ps collisions similar to those for electrons (cf. [15] and the present work) is consistent with the hypothesis that the scattering of Ps is dominated by that of its electron. We note here that a quasifree electron scattering model has been previously applied to the description of resonant transfer and excitation in energetic ion-atom collision (see, e.g., [37]) and Rydberg-atom-neutral-molecule scattering [38]. In the present case, Ps appears to be distorted in the collision so that its electron is on average closer to the target than its positron. Such an effect was initially proposed by Sarkadi [39] to interpret an energy asymmetry, predicted by a classical trajectory Monte Carlo (CTMC) calculation, between the residual electron and positron following the fragmentation of Ps in a collision with helium. Exchange is not included in this approach, the only forces being Coulombic. However, a similar asymmetry arose in the quantal impulse approximation of Starrett et al. [17].

Within a picture where its electron dominates its scattering, Ps itself may be replaced by a flux of electrons of average velocity equal to that of the Ps center of mass and $Q_{\mathrm{T}}^{\mathrm{Ps}}$ may expressed as a weighted average of $Q_{\mathrm{T}}^{-}$over the velocity of the beam and that of the electron in Ps. Mathematically, $Q_{\mathrm{T}}^{\mathrm{Ps}}$ is obtained as a convolution of $Q_{\mathrm{T}}^{-}$by the Compton profile of the electron in Ps and the velocity distribution of the beam. The Compton profile $J\left(p_{z}\right)$ of the electron in Ps, described by a probability density $\left|\psi_{P_{s}}\left(p_{x}, p_{y}, p_{z}\right)\right|^{2}$, is the projection of the probability density along the scattering vector [40],

$$
J\left(p_{z}\right)=\int_{-\infty}^{\infty} \int_{-\infty}^{\infty} d p_{x} d p_{y}\left|\psi_{P s}\left(p_{x}, p_{y}, p_{z}\right)\right|^{2} .
$$

For isotropic systems, this reduces to integration over one dimension [40]

$$
J\left(p_{z}\right)=\frac{1}{2} \int_{\left|p_{z}\right|}^{\infty} d p \frac{1}{p} \rho(p),
$$

where $\rho(p)=4 \pi p^{2}\left|\psi_{P s}(p)\right|^{2}$ is the radial momentum distribution. For Ps in its ground state, $\rho(p)$ is given by [41]

$$
\rho(p)=\frac{32}{\pi p_{0}^{3}} p^{2}\left(\frac{p^{2}}{p_{0}^{2}}+1\right)^{-4},
$$

where $p_{0}^{2}=2 \mu\left|E_{b}\right|$, with $\left|E_{b}\right|$ the binding energy of Ps. The Compton profile of Eq. (2), with $\rho(p)$ given by Eq. (3), may be integrated analytically to yield

$$
J\left(p_{z}\right)=\frac{8}{3 \pi} \frac{p_{0}^{5}}{\left(p_{0}^{2}+p_{z}^{2}\right)^{3}} .
$$

If the kinetic energy of the beam is written as $T=\frac{1}{2} m_{P s} v_{P S}^{2}$, then $\Delta T=m_{P s} v_{P s} \Delta v$ and so the width $\sigma$ (standard deviation) of the velocity distribution is

$$
\sigma=\frac{\Delta T}{4 m_{e} v_{0} \sqrt{2 \ln (2)}},
$$

where $m_{\mathrm{Ps}}\left(m_{e}\right)$ is the mass of Ps $\left(e^{ \pm}\right)$and $v_{0}=v_{\mathrm{Ps}}$ is the velocity of the beam.

We carried out such a convolution for both $\mathrm{N}_{2}$ and $\mathrm{CO}_{2}$. For ground-state Ps, we found this to smear out the resonance peaks in the total cross sections, primarily due to the relatively broad Compton profile of the Ps electron. This implies that the model may be valid only if a narrower Compton profile applies, as in the case of a highly distorted (or virtually excited) Ps. Support for this conjecture comes from a consideration of the energies at which the resonant peaks for Ps and electron impact occur. In Fig. 2 the Ps peak in $Q_{\mathrm{T}}^{\mathrm{Ps}}$ for both targets is shifted with respect to the electron peak $Q_{\mathrm{T}}^{-}$towards higher energies. We interpret this shift $\Delta E_{\text {res }}=E_{\text {res }}^{\mathrm{Ps}}-E_{\text {res }}^{-}$(i.e., the energy difference between the resonance peaks for the two projectiles) as corresponding to the energy expended in distorting the Ps atom in the collision.

The net resonance peaks for both Ps and the convoluted electron-impact data have been determined by fitting the nonresonant part of each cross section to a smooth function and subtracting it from the total peak (see, e.g., [43]). By fitting a Gaussian function to the resonance peak for Ps impact and comparing the fit to the $e^{-}$resonance peak [42], for $\mathrm{CO}_{2}$ we obtain $\Delta E_{\text {res }}=(6.9 \pm 0.1) \mathrm{eV}$, implying an effective binding energy $E_{b}=6.8 \mathrm{eV}-\Delta E_{\text {res }}$ of the virtually excited Ps of zero within errors. For $\mathrm{N}_{2}$, comparing the position of the Ps peak to that of the mean of the two $e^{-}$resonance peaks (at $\sim 2.2$ and $\sim 2.4 \mathrm{eV}$ [33]), we obtain $\Delta E_{\text {res }}=(4.0 \pm 0.3)$, yielding $E_{b}=(2.8 \pm 0.3) \mathrm{eV}$.

Considering that the width of the Compton profile is determined primarily by $E_{b}$, we estimate the profile of the excited Ps by scaling the ground-state distribution accordingly. 


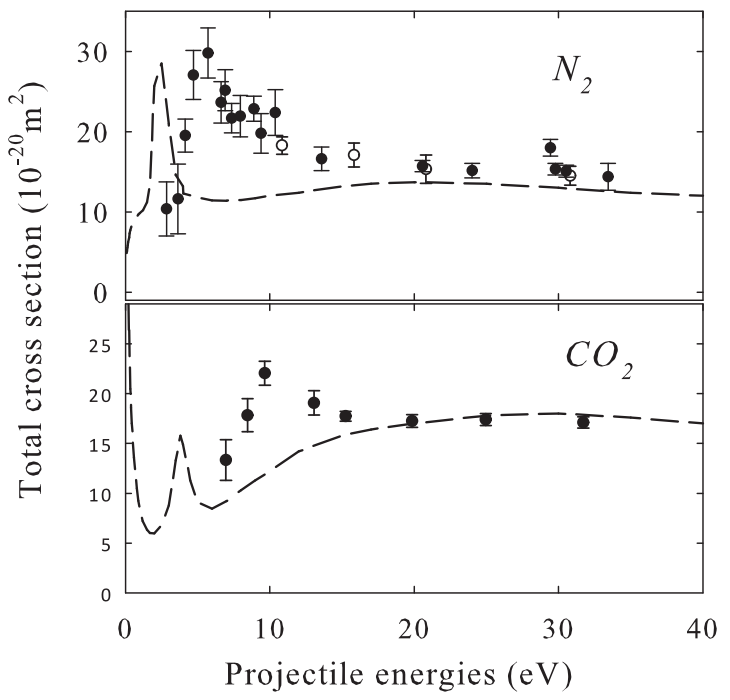

FIG. 2. Total cross sections for $\mathrm{N}_{2}$ and $\mathrm{CO}_{2}$ versus energy for Ps and electron collisions. For $\mathrm{N}_{2}$, the $\bullet$ represent the $Q_{\mathrm{T}}^{\mathrm{Ps}}$ measurements from the present work and the $\circ$ those from [16], while — — — shows $Q_{\mathrm{T}}^{-}$from [33]. For $\mathrm{CO}_{2}$, the $\bullet$ represent the $Q_{\mathrm{T}}^{\mathrm{Ps}}$ measurements from [15] and - - shows $Q_{\mathrm{T}}^{-}$from [42].

This approximation provides a degree of self-consistency via the constraint that the magnitude and width of the convoluted electron-impact peak should agree well with those of the measured Ps peak.

The results of convolutions for $\mathrm{CO}_{2}$ and $\mathrm{N}_{2}$ are shown in Fig. 3 with $\Delta E_{\text {res }}\left(\mathrm{CO}_{2}\right)=0 \mathrm{eV}$ and $\Delta E_{\text {res }}\left(\mathrm{N}_{2}\right)=2.8 \mathrm{eV}$. The convoluted $Q_{\mathrm{T}}^{-}$are compared with $Q_{\mathrm{T}}^{\mathrm{Ps}}$, the latter shifted to lower energies by the appropriate $\Delta E_{\text {res }}$. For $\mathrm{CO}_{2}$, there is

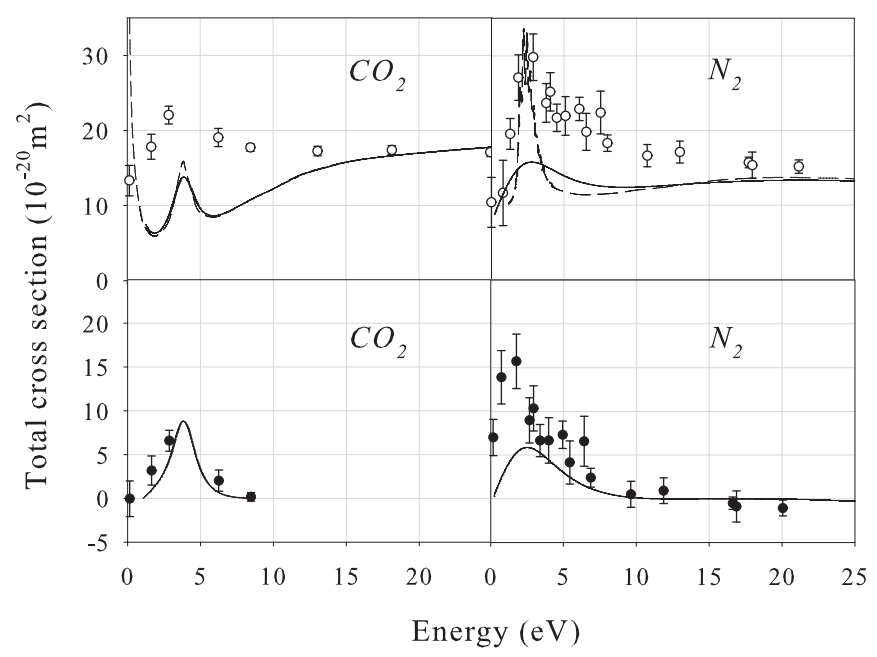

FIG. 3. Comparison of $Q_{\mathrm{T}}^{\mathrm{Ps}}$ with the convoluted $Q_{\mathrm{T}}^{-}$for collisions with $\mathrm{CO}_{2}$ (left) and $\mathrm{N}_{2}$ (right). In the top panels open circles show $Q_{\mathrm{T}}^{\mathrm{Ps}}$ measurements for $\mathrm{CO}_{2}$ from [15] and for $\mathrm{N}_{2}$ from [16] and the present work; dashed lines show $Q_{\mathrm{T}}^{-}$measurements for $\mathrm{CO}_{2}$ from [42] and for $\mathrm{N}_{2}$ from [33]; the solid line shows convoluted $Q_{\mathrm{T}}^{-}$. Shown in the bottom panels are the convoluted (lines) and measured (circles) resonance peaks for Ps impact after subtracting the nonresonant contribution. For each target, the Ps data have been shifted to lower energies by the appropriate $\Delta E_{\text {res }}$.

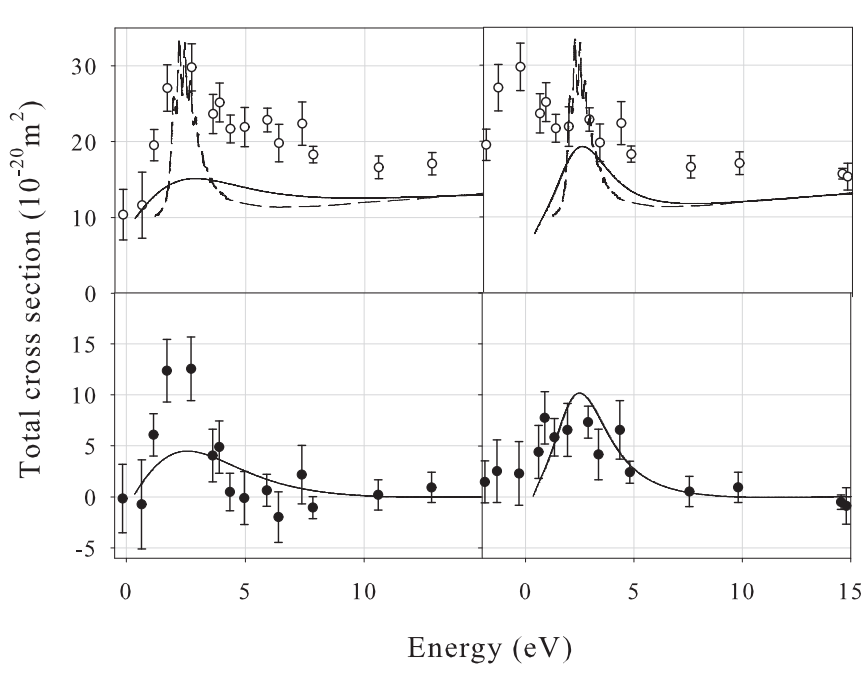

FIG. 4. Comparison of the convoluted $Q_{\mathrm{T}}^{-}$with $Q_{\mathrm{T}}^{\text {Ps }}$ for collision with $\mathrm{N}_{2}$ with respect to the peak at $\sim 5 \mathrm{eV}$ (left) and $\sim 8 \mathrm{eV}$ (right). The legend is the same as for Fig. 3.

a reasonable correspondence between the Ps and convoluted electron peaks, but much less so in the case of $\mathrm{N}_{2}$. These net cross sections are absolute, i.e., not normalized at their maxima.

We performed a further analysis for $\mathrm{N}_{2}$, noting the structure in $Q_{\mathrm{T}}^{\mathrm{Ps}}$ around 8-10 eV apparent in Fig. 2, with convolutions treating the Ps peak as overlapping peaks, one at $E_{\mathrm{Ps}} \sim 5 \mathrm{eV}$ and the second centered at $\sim 8 \mathrm{eV}$. The results of this are shown in Fig. 4. Here we calculated the goodness of fit of the net Ps peak to the net convoluted electron peak. For the peak at $\sim 5 \mathrm{eV}$ the reduced $\chi^{2}$ was 2.0 and for the peak at $\sim 8 \mathrm{eV}$ it was 1.1 , suggesting that the peak at $\sim 8 \mathrm{eV}$ reflects the resonance in the electron cross section, while the additional structure at $\sim 5 \mathrm{eV}$ may be linked intrinsically to Ps scattering. It is noted that structure around $5 \mathrm{eV}$, close to the excitation energy to $n=2$, has also been observed in the Ps-Ar and Ps-Xe total cross sections [11].

Encouraged by the above results, we have used a CTMC calculation to monitor the time evolution of the positions and velocities of the constituent particles in Ps during collision. Although in the present approach CTMC cannot account for resonance formation, it is expected to give a fair description of the distortion of Ps, especially in the incoming phase of the collision. For this purpose, we considered the scattering of Ps from a completely screened Coulomb potential of a neutral atomic target [44]. As the target we chose one of the atoms of $\mathrm{CO}_{2}$, namely, oxygen, and made the calculations at $v_{0}=0.528$ a.u., the velocity at which the resonant peak occurs in $Q_{\mathrm{T}}^{-}$for $\mathrm{CO}_{2}$ [42]. As a further simplification, the oxygen atom is considered to be structureless, so the many-particle problem is reduced to a three-body problem. For the calculations, we used the computer code described in [45].

The procedure is similar to that employed by Sarkadi [39] for the description of the fragmentation of Ps in a collision with helium. In the present case, we stopped the integration at selected times from the closest approach of the collision partners ( $t=0$ by definition). Figure 5 shows 

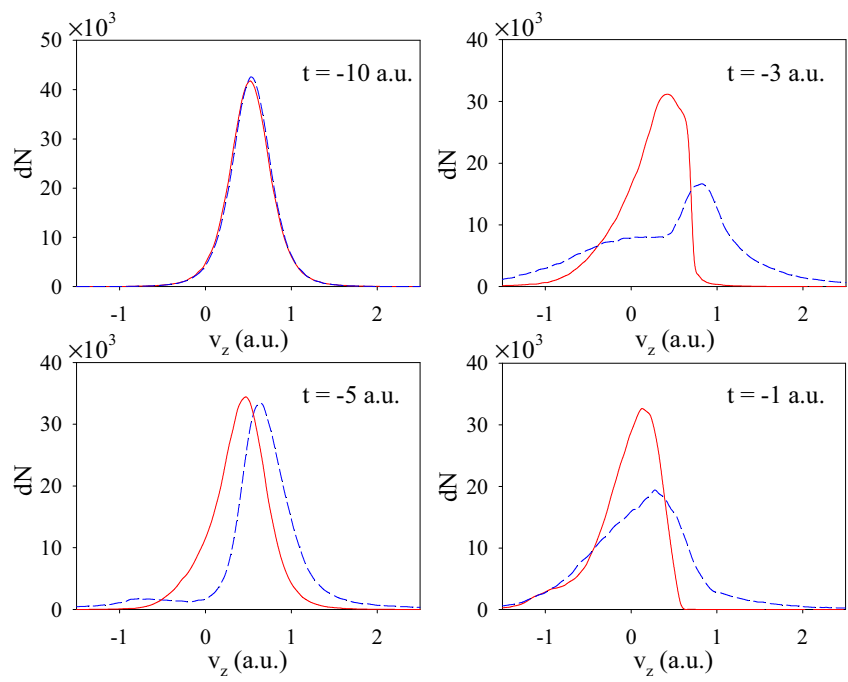

FIG. 5. Time evolution of the longitudinal velocity distribution of the electron (dashed curve) and the positron (solid curve) predicted by CTMC for Ps on oxygen collisions at the resonance velocity $v_{0}=0.528$ a.u. (see the text).

the results for the longitudinal velocity $v_{z}$ distribution of the electron and the positron. We considered only head-on (zero-impact-parameter) collisions. As illustrated, there is only a slight change in the positron velocity distributions, while that for the electron at first shifts to higher velocities and then broadens. In Fig. 6 the polarization of the incoming Ps by the target nucleus is evident in the position distribution of the electron and the positron, the positron being kept farther away from the target.

The distortion of Ps as outlined above is also consistent with recent theoretical descriptions [46-50] employed to provide an explanation of the similarity between the cross sections for positronium scattering and electron scattering above the Ps breakup threshold. The assumption of negligible binding energy of Ps is certainly warranted for virtually excited Ps, the extent of which our method quantifies. The nature of the observed resonances may in fact be not too dissimilar from that predicted by quantum theories for the simpler Ps $+\mathrm{H}$ system [21-24]. The interaction between the quasifree electron and the molecule may lead to an intermediate negative-ion resonance state of the target molecule; in other words, the $e^{-}$ is captured transiently by the target and the positron is left with little energy, in accordance with the definition of $\Delta E_{\text {res. }}$ The resulting complex thus forms a transient negative center around which the positron may also be captured. The lifetime of the
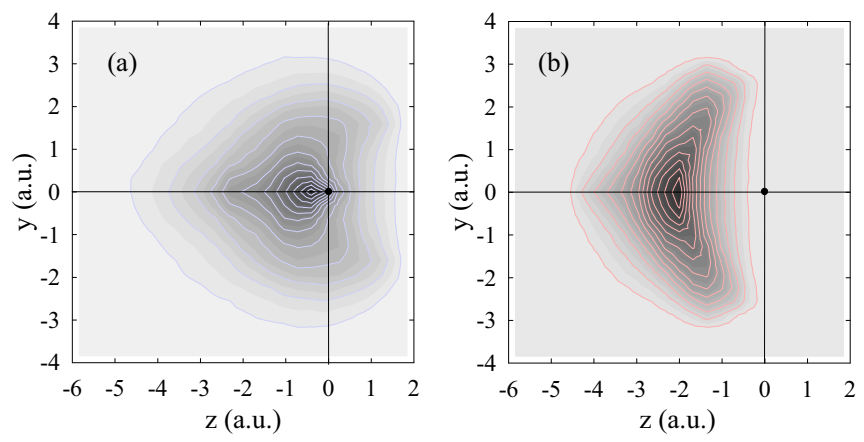

FIG. 6. Contour plot of the distribution of the $(y, z)$ coordinates of the (a) electrons and (b) positrons in collisions of Ps with oxygen atoms at time $t=-1$ a.u.

resonance $\left(\simeq 10^{-16} \mathrm{~s}\right)$ is much shorter than the lifetime of $o$-Ps and even of spin-singlet $\operatorname{Ps}\left(\simeq 10^{-10} \mathrm{~s}\right)$ and so, assuming pickoff quenching to be negligible, the $e^{+}$might survive and the Ps atom might reemerge from the collision. This may happen since the attractive center for the positron ceases to exist upon decay of the electron resonance so that the positron and the electron are released at essentially the same time.

In summary, we have measured the total cross section of Ps $+\mathrm{N}_{2}$ and have found it to display significant structure in the vicinity of that in the corresponding electron data. We have analyzed the present and previous data $[15,16]$ by ascribing the energy shifts between the resonance positions for the two projectiles to the energy expended in distorting the Ps. Hence, the reduced binding energy of Ps was used to estimate its Compton profile, which we used to convolute the electron data before comparing them with the Ps measurements. The quantitative consistency between the two explains the physical origin of Ps being resonantly scattered like a quasifree electron; the positron, which in the initial phase of the collision appears to play a spectator role, may (once the electron resonance is formed) itself participate by binding resonantly to the ensuing temporary negative ion. This description of the nature of Ps resonances is in accord with long-standing theoretical predictions [19-23].

We are grateful to James Walters, Adam Williams, Jonathan Tennyson, and Ilya Fabrikant for valuable discussions, to EPSRC (Grants No. EP/J003980/1 and No. EP/P009395/1) and the National Scientific Research Foundation (OTKA, Grant No. K109440) for financial support, and to John Dumper and Rafid Jawad for expert technical assistance.
[1] C. Winkler, R. Diehl, P. Ubertini, and J. Wilms, Space Sci. Rev. 161, 149 (2011).

[2] S. Polarz and B. Smarsly, J. Nanosci. Nanotechnol. 2, 581 (2002).

[3] J. Laverock, S. B. Dugdale, M. A. Alam, M. V. Roussenova, J. R. Wensley, J. Kwiatkowska, and N. Shiotani, Phys. Rev. Lett. 105, 236401 (2010).

[4] S. R. Cherry, Phys. Med. Biol. 49, R13 (2004).
[5] Radiation Damage in Biomolecular Systems, edited by G. Garcia and M. C. Fuss (Springer Science+Business Media, New York, 2012).

[6] D. B. Cassidy, T. H. Hisakado, H. W. K. Tom, and A. P. Mills, Phys. Rev. Lett. 106, 173401 (2011).

[7] H. K. Avetissian, A. K. Avetissian, and G. F. Mkrtchian, Phys. Rev. Lett. 113, 023904 (2014). 
[8] D. B. Cassidy, T. H. Hisakado, H. W. K. Tom, and A. P. Mills, Phys. Rev. Lett. 108, 043401 (2012).

[9] A. Deller, A. M. Alonso, B. S. Cooper, S. D. Hogan, and D. B. Cassidy, Phys. Rev. Lett. 117, 073202 (2016).

[10] G. Laricchia and H. R. J. Walters, Riv. Nuovo Cimento 35, 305 (2012).

[11] S. J. Brawley, S. E. Fayer, M. Shipman, and G. Laricchia, Phys. Rev. Lett. 115, 223201 (2015).

[12] N. Zafar, G. Laricchia, M. Charlton, and A. Garner, Phys. Rev. Lett. 76, 1595 (1996).

[13] A. Garner, G. Laricchia, and A. Özen, J. Phys. B 29, 5961 (1996).

[14] S. Armitage and G. Laricchia, Nucl. Instrum. Methods Phys. Res. Sect. B 192, 67 (2002).

[15] S. J. Brawley, A. I. Williams, M. Shipman, and G. Laricchia, Phys. Rev. Lett. 105, 263401 (2010).

[16] S. Brawley, S. Armitage, J. Beale, D. Leslie, A. Williams, and G. Laricchia, Science 330, 789 (2010).

[17] C. Starrett, M. T. McAlinden, and H. R. J. Walters, Phys. Rev. A 72, 012508 (2005).

[18] H. R. J. Walters, Science 330, 762 (2010).

[19] R. J. Drachman and S. K. Houston, Phys. Rev. A 12, 885 (1975).

[20] R. J. Drachman and S. K. Houston, Phys. Rev. A 14, 894 (1976).

[21] R. J. Drachman, Phys. Rev. A 19, 1900 (1979).

[22] J. E. Blackwood, M. T. McAlinden, and H. R. J. Walters, Phys. Rev. A 65, 030502 (2002).

[23] H. R. J. Walters, A. C. H. Yu, S. Sahoo, and S. Gilmore, Nucl. Instrum. Methods Phys. Res. Sect. B 221, 149 (2004).

[24] Y. K. Ho, Phys. Rev. A 41, 68 (1990).

[25] M. Shipman, S. J. Brawley, L. Sarkadi, and G. Laricchia, Eur. Phys. J. D 68, 75 (2014).

[26] A. Özen, A. Garner, and G. Laricchia, Nucl. Instrum. Methods Phys. Res. Sect. B 171, 172 (2000).

[27] M. Shipman, S. Armitage, J. Beale, S. J. Brawley, S. E. Fayer, A. J. Garner, D. E. Leslie, P. Van Reeth, and G. Laricchia, Phys. Rev. Lett. 115, 033401 (2015).
[28] A. J. Garner and G. Laricchia, Can. J. Phys. 74, 518 (1996).

[29] G. Laricchia, N. Zafar, M. Charlton, and T. Griffith, Hyperfine Int. 73, 133 (1992).

[30] A. Garner, A. Özen, and G. Laricchia, Nucl. Instrum. Methods Phys. Res. Sect. B 143, 155 (1998).

[31] G. Laricchia, S. Armitage, and D. Leslie, Nucl. Instrum. Methods Phys. Res. Sect. B 221, 60 (2004).

[32] M. Shipman, S. J. Brawley, D. E. Leslie, S. Armitage, and G. Laricchia, Eur. Phys. J. D 66, 96 (2012).

[33] Y. Itikawa, J. Phys. Chem. Ref. Data 35, 31 (2006).

[34] L. Christophorou, J. Olthoff, and M. Rao, J. Phys. Chem. Ref. Data 25, 1341 (1996).

[35] O. Sueoka, S. Mori, and A. Hamada, J. Phys. B 27, 1453 (1994).

[36] A. Zecca, L. Chiari, A. Sarkar, and M. Brunger, New J. Phys. 13, 115001 (2011).

[37] D. Brandt, Phys. Rev. A 27, 1314 (1983).

[38] P. M. Koch, Phys. Rev. Lett. 43, 432 (1979).

[39] L. Sarkadi, Phys. Rev. A 68, 032706 (2003).

[40] M. Cooper, Rep. Prog. Phys. 48, 415 (1985).

[41] B. H. Bransden and C. J. Joachain, Physics of Atoms and Molecules, 2nd ed. (Prentice Hall, Englewood Cliffs, 2002), pp. 1014-1015.

[42] Y. Itikawa, J. Phys. Chem. Ref. Data 31, 749 (2002).

[43] G. C. King, Adv. At. Mol. Opt. Phys. 60, 1 (2011).

[44] A. Green, D. L. Sellin, and A. S. Zachor, Phys. Rev. 184, 1 (1969).

[45] B. Sulik and K. Tókési, Adv. Quantum Chem. 52, 253 (2007).

[46] I. I. Fabrikant and G. F. Gribakin, Phys. Rev. Lett. 112, 243201 (2014).

[47] I. I. Fabrikant and G. F. Gribakin, Phys. Rev. A 90, 052717 (2014).

[48] A. R. Swann, J. A. Ludlow, and G. F. Gribakin, Phys. Rev. A 92, 012505 (2015).

[49] R. S. Wilde and I. I. Fabrikant, Phys. Rev. A 92, 032708 (2015).

[50] G. F. Gribakin, A. R. Swann, R. S. Wilde, and I. I. Fabrikant, J. Phys. B 49, 064004 (2016). 